\title{
Increased cancer risk in patients undergoing dialysis: a population-based cohort study in North-Eastern Italy
}

\author{
Martina Taborelli ${ }^{1 *}$ DD, Federica Toffolutti ${ }^{1}$, Stefania Del Zotto ${ }^{1}$, Elena Clagnan² ${ }^{2}$ Lucrezia Furian ${ }^{3}$, Pierluca Piselli ${ }^{4}$, \\ Franco Citterio ${ }^{5}$, Loris Zanier ${ }^{2}$, Giuliano Boscutti ${ }^{6}$, Diego Serraino ${ }^{1}$ and for the Italian Transplant \& Cancer Cohort \\ Study
}

\begin{abstract}
Background: In southern Europe, the risk of cancer in patients with end-stage kidney disease receiving dialysis has not been well quantified. The aim of this study was to assess the overall pattern of risk for de novo malignancies (DNMs) among dialysis patients in the Friuli Venezia Giulia region, north-eastern Italy.

Methods: A population-based cohort study among 3407 dialysis patients was conducted through a record linkage between local healthcare databases and the cancer registry (1998-2013). Person-years (PYs) were calculated from 30 days after the date of first dialysis to the date of DNM diagnosis, kidney transplant, death, last follow-up or December 31, 2013, whichever came first. The risk of DNM, as compared to the general population, was estimated using standardized incidence ratios (SIRs) and 95\% confidence intervals (Cls).

Results: During 10,798 PYs, 357 DNMs were diagnosed in 330 dialysis patients. A higher than expected risk of 1.3fold was found for all DNMs combined (95\% Cl: 1.15-1.43). The risk was particularly high in younger dialysis patients $(\mathrm{SIR}=1.88,95 \% \mathrm{Cl}: 1.42-2.45$ for age 40-59 years), and it decreased with age. Moreover, significantly increased DNM risks emerged during the first 3 years since dialysis initiation, especially within the first year ( $\mathrm{SIR}=8.52,95 \% \mathrm{Cl}: 6.89$ 10.41). Elevated excess risks were observed for kidney ( $\mathrm{SIR}=3.18 ; 95 \% \mathrm{Cl}: 2.06-4.69)$, skin non-melanoma ( $\mathrm{SIR}=1.81$, 95\% Cl: 1.46-2.22), oral cavity ( $\mathrm{SIR}=2.42,95 \% \mathrm{Cl}$ : $1.36-4.00$ ), and Kaposi's sarcoma ( $\mathrm{SIR}=10.29,95 \% \mathrm{Cl}$ : 1.25-37.16).
\end{abstract}

Conclusions: The elevated risk for DNM herein documented suggest the need to implement a targeted approach to cancer prevention and control in dialysis patients.

Keywords: Dialysis, Cancer risk, de novo malignancies, End-stage kidney disease, Population-based study, Italy

\section{Background}

Patients receiving renal replacement therapies (RRT) are known to be at higher risk of cancer than the corresponding general population [1-3]. Although some malignancies diagnosed in dialysis patients or after kidney transplant (KT) share similar risk factors (e.g., hepatitis $B$ virus infection for liver cancer), the magnitude and pattern of increased risks substantially vary according to individual and clinical characteristics and to the modality of RRT [4].

\footnotetext{
*Correspondence: mtaborelli@cro.it

${ }^{1}$ Cancer Epidemiology Unit, Centro di Riferimento Oncologico di Aviano (CRO) IRCCS, via Franco Gallini 2, 33081 Aviano, (PN), Italy

Full list of author information is available at the end of the article
}

It is well known that emerging techniques and advances in dialysis technology have led to significant improvements in patients' life span. Due to increased survival, malignant neoplasms have become an increasingly relevant health issue in the dialysis population [5]. Previous studies have provided convincing evidence of an increased risk of certain cancer types, such as kidney, thyroid, and bladder cancer $[1,3,6,7]$, possibly related to side effects of kidney failure, including the prolonged uremic state, the presence of chronic infection and inflammation, a weakened immune system, nutritional deficiencies, and impaired mechanisms of DNA repair [8].

The investigation of the pattern of cancer risk in dialysis patients is crucial for planning primary and secondary

(c) The Author(s). 2019 Open Access This article is distributed under the terms of the Creative Commons Attribution 4.0 International License (http://creativecommons.org/licenses/by/4.0/), which permits unrestricted use, distribution, and 
cancer prevention strategies. The increased risk of cancer in dialysis patients has been well documented in Asia [3, 6], United States [7], Australia/New Zealand [2], and northern Europe [9]. Conversely, less evidence has been accumulated in southern European countries, such as Italy, where end-stage kidney disease (ESKD) patients represent about $0.1 \%$ of the general population [10], and only a small study has been published in the international literature [11].

In this population-based investigation, we assessed the risk and spectrum of de novo malignancies (DNMs) among dialysis patients as compared to the corresponding general population.

\section{Methods}

A population-based cohort study was conducted using information ascertained through a record linkage procedure from three health-related databases, which cover the totality of the population in the Friuli Venezia Giulia region (1,227,000 inhabitants): 1 ) the health information system, which provides personal (e.g., sex, birthdate, residence, and vital status) and medical care data (e.g., hospital discharge, outpatient care, and pharmaceutical health care); 2) the renal registry database, which includes information about patients who underwent at least two dialytic sessions (either peritoneal dialysis or hemodialysis) per week for at least 90 days; 3) the population-based cancer registry, which collects data on all new cases of cancer occurring among the population living in the Friuli Venezia Giulia region. The cancer registry started collecting data on new cancer diagnoses in the resident population in January 1995. For the aims of this analysis, and to guarantee the highest completeness and accuracy of information, data from these three databases were linked only to the concurrently available period, i.e., 1998-2013.

For the aim of this study, the analysis was restricted to dialysis patients residing in the Friuli Venezia Giulia region between 1998 and 2013 and aged 40 years or older at their first dialysis. We focused on dialysis patients aged $\geq 40$ years, because this age group covered $95 \%$ of all patients who had started their first dialysis between 1998 and 2013 and 99\% of all cancer diagnoses in this population. Excluded from the analysis were subjects who met at least one of the following conditions: (i) KT before dialysis $(N=709)$; (ii) residence outside the Friuli Venezia Giulia region during the dialysis, or missing information on residence $(N=113)$; (iii) follow-up shorter than 30 days $(N=22)$. Accordingly to these criteria, 3407 dialysis patients represented our study population.

Cancer diagnoses were coded according to the International Classification of Disease, 9th revision (ICD-9). Cancer diagnoses done by autopsy only (i.e., post-mortem diagnoses; $n=12$ ) were not considered in this study because of lack of completeness of this information during the whole registration period. Multiple primary tumours were included in the site-specific analyses, while for patients diagnosed with more than one DNM within the same ICD-9 group (e.g., colon, rectum, and anus ICD-9 codes: 153-154), only the first one was considered.

Person-years at risk were calculated from 30 days after the date of first dialysis to the date of DNM diagnosis, to the date of $\mathrm{KT}$, to the date of death, to end of follow-up or to December 31, 2013 (i.e., censored cases), whichever came first. We excluded from the analysis the first 30 days of follow-up in order to reduce prevalent DNMs. After a DNM diagnosis, patients did not contribute any longer to person-time at risk for that specific cancer site/type. Conversely, those patients continued to be at risk of other cancer sites/types in the specific analyses. Patients with a history of cancer prior to their first dialysis were not considered eligible to contribute to person-time at risk for that specific cancer site/type.

The cumulative cancer incidence by time since first dialysis and cancer site/type was estimated using a competing risk approach with nonparametric estimators [12], taking into account death as a competing event. Standardized incidence ratios (SIRs) were estimated as the ratio between the observed number of cancer cases among the 3407 cohort members and the expected number of cancer cases among the general population of the Friuli Venezia Giulia region. According to a standard procedure [13-15], the expected number of cases was computed by multiplying the person-years at risk among the 3407 dialysis patients with the sex- and age-adjusted cancer incidence rates in the general population of the Friuli Venezia Giulia region derived from the local population-based cancer registry. Incidence rates for the general population were computed using, as numerator, the observed number of cancer cases, and, as denominator, the average resident population of same sex and age, as a proxy of person-years at risk.

SIRs for all cancers combined were also estimated according to sex, age at first dialysis $(40-59,60-74, \geq 75$ years), calendar period at first dialysis (1998-2001, 2002-2005, 2006-2009, 2010-2013), follow-up time $(<1,1$ to $<2,2$ to $<3,3$ to $<5, \geq 5$ years), and dialysis modality (haemodialysis, peritoneal dialysis, both haemodialysis and peritoneal dialysis). Corresponding 95\% confidence intervals (CIs) for SIRs were computed assuming a Poisson distribution [13].

\section{Results}

The 3407 dialysis patients were followed up for a total of 10,798 person-years (Table 1), with a median follow-up time of 2.3 years (interquartile range, IQR: $1.0-4.5$ years). Most of these 3407 patients were males (62.4\%), aged between 60 and 74 years at first dialysis (42.6\%; median 
Table 1 Distribution of 3407 dialysis patients by selected characteristics. Friuli Venezia Giulia, north-eastern Italy, 1998-2013

\begin{tabular}{|c|c|}
\hline & $\begin{array}{l}\text { All patients } \\
\text { No. (\%) }\end{array}$ \\
\hline \multicolumn{2}{|l|}{ Sex } \\
\hline Male & $2126(62.4)$ \\
\hline Female & $1281(37.6)$ \\
\hline \multicolumn{2}{|l|}{ Age at first dialysis (years) } \\
\hline $40-59$ & $746(21.9)$ \\
\hline $60-74$ & $1452(42.6)$ \\
\hline$\geq 75$ & $1209(35.5)$ \\
\hline Median (Interquartile Range) & $70(61-77)$ \\
\hline \multicolumn{2}{|l|}{ Calendar year at first dialysis } \\
\hline 1998-2001 & $1208(35.5)$ \\
\hline $2002-2005$ & $674(19.8)$ \\
\hline $2006-2009$ & $802(23.5)$ \\
\hline 2010-2013 & $723(21.2)$ \\
\hline \multicolumn{2}{|l|}{ Dialysis modality } \\
\hline Haemodialysis & $2858(83.9)$ \\
\hline Peritoneal dialysis & $201(5.9)$ \\
\hline Both haemodialysis and peritoneal dialysis & $348(10.2)$ \\
\hline \multicolumn{2}{|l|}{ Follow-up (years) } \\
\hline$<1$ & $867(25.4)$ \\
\hline $1-<2$ & $674(19.8)$ \\
\hline $2-<3$ & $539(15.8)$ \\
\hline $3-<5$ & $632(18.6)$ \\
\hline$\geq 5$ & $695(20.4)$ \\
\hline Median (Interquartile Range) & $2.3(1.0-4.5)$ \\
\hline Total person-years & 10,798 \\
\hline \multicolumn{2}{|l|}{ Patient with de novo malignancies during dialysis } \\
\hline No & $3077(90.3)$ \\
\hline Yes & $330(9.7)$ \\
\hline \multicolumn{2}{|l|}{ No. of de novo malignancies during dialysis } \\
\hline 1 & $305(92.4)$ \\
\hline 2 & $23(7.0)$ \\
\hline 3 & $2(0.6)$ \\
\hline
\end{tabular}

age: 70 years; IQR: $61-77$ years), and were treated with haemodialysis $(83.9 \%)$. More than one third underwent dialysis between 1998 and 2001 (35.5\%). During the period of observation, 357 DNMs were diagnosed in 330 dialysis patients (9.7\%) of whom, 25 had more than one DNM.

The cumulative incidence of all DNMs combined steadily increased with time since beginning of dialysis, reaching $9.8 \%$ after 5 years and $13.9 \%$ after 10 years (Fig. 1). A similar pattern emerged after the exclusion of non-melanoma skin cancers (NMSC), with 5- and 10 -year cumulative risks equal to $8.9 \%$ and $12.8 \%$, respectively.

Starting at 40-44 years, the age-specific incidence rates for de novo malignancies observed among dialysis patients steadily increased from 670 per 100,000 PYs to 3965 per 100,000 PYs among those aged 85 years and older (Fig. 2). In dialysis patients, incidence rates were consistently higher than those in the general population of the Friuli Venezia Giulia region, even though these differences decreased with increasing age.

Considering all DNMs combined, a 1.3-fold higher risk was found in dialysis patients than in the corresponding general population (95\% CI: 1.15-1.43) (Table 2). The subgroup analysis showed similar SIRs for both sexes $(\mathrm{SIR}=1.29,95 \% \mathrm{CI}: 1.14-1.46$ in males and $\mathrm{SIR}=1.27$, 95\% CI: $1.01-1.57$ in females). Furthermore, the risk of DNM was particularly high in younger dialysis patients (SIR $=1.88$, 95\% CI: $1.42-2.45$ for age $40-59$ years) and decreased with age. Elevated excess risks were also observed during the first 3 years since dialysis initiation, especially within the first year (SIR $=8.52$, 95\% CI: 6.8910.41). When comparing the risk of DNM according to the modality of dialysis, the SIRs were 1.26 (95\% CI: $1.12-1.42)$ in patients treated with haemodialysis and 1.78 (95\% CI: 0.95-3.04; based on 13 observed cases of DNMs) in those treated with peritoneal dialysis.

Table 3 lists the SIRs for specific DNMs with at least two observed cases. When considering all cancer types/ sites other than NMSC, a 1.2-fold elevated cancer risk emerged, as compared to the general population (95\% CI:1.03-1.32). Statistically significant increased risks were documented for NMSC $(\mathrm{SIR}=1.81$; 95\% CI: $1.46-$ 2.22 ), cancers of kidney ( $\mathrm{SIR}=3.18$; 95\% CI: 2.06-4.69), oral cavity (SIR $=2.42,95 \%$ CI: $1.36-4.00)$, and Kaposi's sarcoma (SIR $=10.29$, 95\% CI: $1.25-37.16)$.

\section{Discussion}

The findings from this population-based cohort study conducted in north-eastern Italy showed that the cumulative cancer risk of dialysis patients reached about $14 \%$ after 10 years of follow-up. This observation corresponded to an overall 1.3-fold higher risk of DNM, as compared to the general population. Significantly increased cancer risks were seen for younger patients, and within the first three years of dialysis, as well as for several cancer sites/types, including kidney, oral cavity, NMSC, and Kaposi's sarcoma.

Several studies have looked at the relationship between dialysis and cancer incidence, yielding overall estimates of excess cancer risk that were super-imposable to those reported in the present investigation [2, 3, 14]. Notably, results from a recent meta-analysis of population-based cohort studies showed that, in comparison with the 


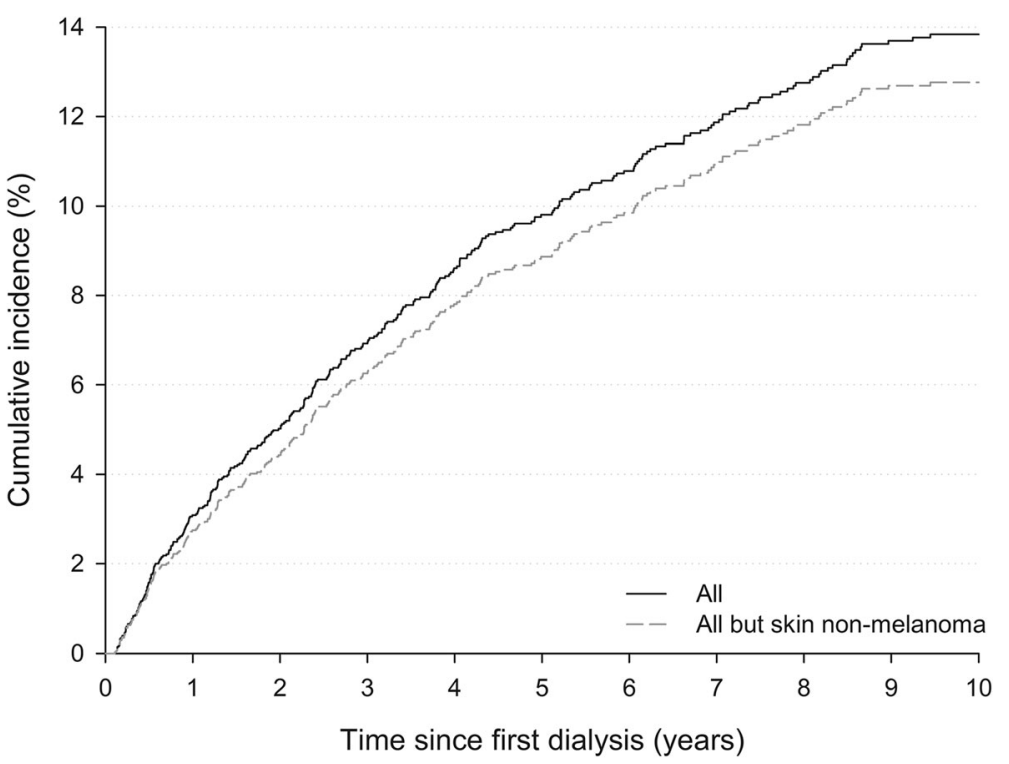

Fig. 1 Cumulative cancer incidence by time since first dialysis. Friuli Venezia Giulia, north-eastern Italy, 1998-2013

general population, the pooled SIRs for DNM in dialysis patients including or excluding NMSC were 1.40 (95\% CI: $1.36-1.45)$ and 1.35 (95\% CI: 1.23-1.50), respectively [16]. To the best of our knowledge, only one study, carried out in Denmark, reported no evidence of a statistically significant increased risk of cancer after excluding NMSC (SIR $=1.16,95 \%$ CI: 0.92-1.45) [15].

In the subgroup analyses, we found increased cancer risks that were in line with previous studies carried out in other industrialized countries [1, 3, 17]. Our results showed that the excess risk of cancer was higher in younger dialysis patients, while it declined with increasing age up to 75 years. Accordingly, a multicenter cohort study reported a high excess risk in the youngest age group (i.e., $<45$ years at dialysis), which became lower in patients older than 65 years [14]. This age-related pattern may have different explanations. Firstly, the enhanced risk in younger patients is largely attributable to the low cancer incidence rates among young people in the general population. Secondly, younger patients may be affected by more serious virus-related malignancies against which their immune defenses, already compromised because of

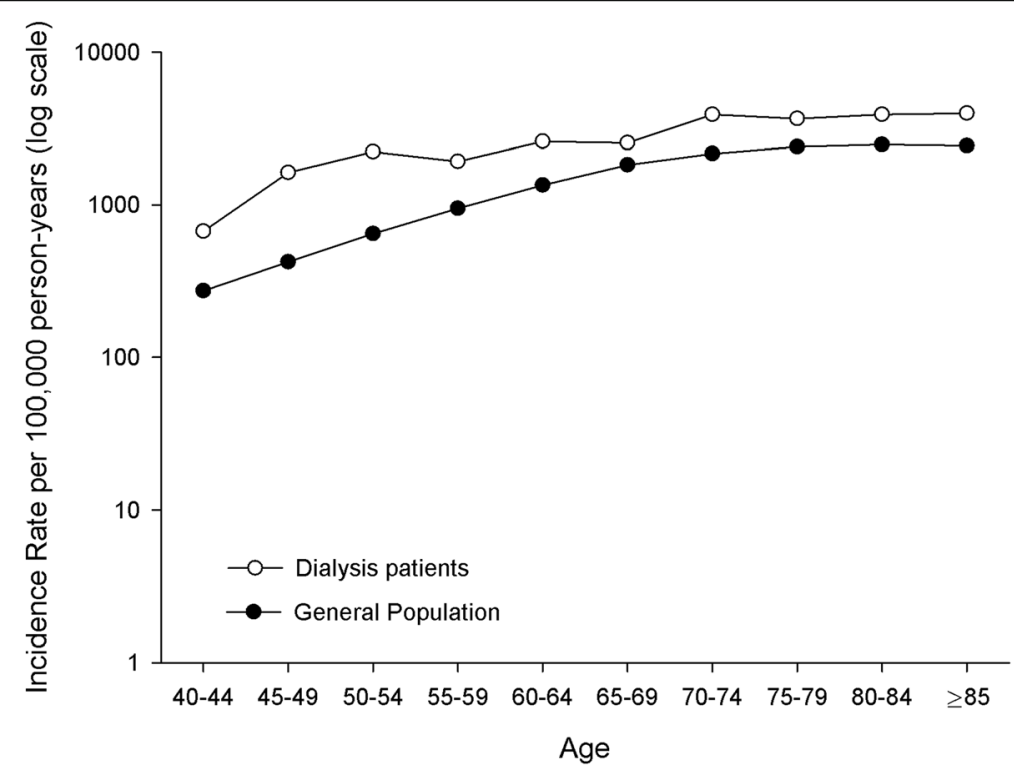

Fig. 2 Age-specific incidence rates for de novo malignancies observed in dialysis patients and in the general population of Friuli Venezia Giulia region. Friuli Venezia Giulia, north-eastern Italy, 1998-2013 
Table 2 Standardized incidence ratios (SIRs) and 95\% confidence intervals (CIs) in dialysis patients for all de novo malignancies (DNMs), by selected subgroups. Friuli Venezia Giulia, north-eastern Italy, 1998-2013

\begin{tabular}{|c|c|c|c|}
\hline & \multicolumn{3}{|c|}{ Total $^{a}$} \\
\hline & Obs. & Exp. & $\operatorname{SIR}(95 \% \mathrm{Cl})$ \\
\hline All & 330 & 256.6 & $1.29(1.15-1.43)$ \\
\hline \multicolumn{4}{|l|}{ Sex } \\
\hline Male & 246 & 190.5 & $1.29(1.14-1.46)$ \\
\hline Female & 84 & 66.2 & $1.27(1.01-1.57)$ \\
\hline \multicolumn{4}{|l|}{ Age at first dialysis (years) } \\
\hline $40-59$ & 56 & 29.7 & $1.88(1.42-2.45)$ \\
\hline $60-74$ & 176 & 131.0 & $1.34(1.15-1.57)$ \\
\hline$\geq 75$ & 98 & 95.9 & $1.02(0.83-1.24)$ \\
\hline \multicolumn{4}{|l|}{ Calendar year at first dialysis } \\
\hline 1998-2001 & 136 & 106.2 & $1.28(1.07-1.52)$ \\
\hline $2002-2005$ & 79 & 63.3 & $1.25(0.99-1.56)$ \\
\hline 2006-2009 & 76 & 60.6 & $1.25(0.99-1.58)$ \\
\hline 2010-2013 & 39 & 26.6 & $1.47(1.04-2.00)$ \\
\hline \multicolumn{4}{|l|}{ Dialysis modality } \\
\hline Haemodialysis & 279 & 221.7 & $1.26(1.12-1.42)$ \\
\hline Peritoneal dialysis & 13 & 7.3 & $1.78(0.95-3.04)$ \\
\hline Both haemodialysis and peritoneal dialysis & 38 & 27.7 & $1.37(0.97-1.88)$ \\
\hline \multicolumn{4}{|l|}{ Follow-up (years) } \\
\hline$<1$ & 95 & 11.2 & $8.52(6.89-10.41)$ \\
\hline $1-<2$ & 52 & 24.2 & $2.15(1.61-2.82)$ \\
\hline $2-<3$ & 48 & 32.2 & $1.49(1.10-1.97)$ \\
\hline $3-<5$ & 56 & 62.0 & $0.90(0.68-1.17)$ \\
\hline$\geq 5$ & 79 & 127.1 & $0.62(0.49-0.77)$ \\
\hline
\end{tabular}

${ }^{\mathrm{a}}$ For patients diagnosed with more than one DNM, only the first one was considered; Obs., observed number of cancer cases; Exp., expected number of cancer cases

the uremic state, tend to be lacking, compared to older people $[3,18]$. Thirdly, the presence of ESKD, comorbidities and frailty could lead elderly dialysis patients to die from other chronic illnesses, such as cardiovascular diseases, before the development of cancer. Thus, the discrepancy in cancer risk may disappear with increasing age.

We observed an elevated cancer risk among both male and female patients. A large international collaborative study reported that the overall risk for cancer was higher in females than in males during dialysis [1], but other investigations have reported contradictory findings [19].

Subgroup analysis by follow-up time showed that the risk of DNM was highest within the first year after dialysis initiation and decreased proportionally with the duration of dialysis. Similar figures were found in other investigations [1, 3, 17], with the exception of a large-scale cohort study, which reported the highest cancer risk after 4 years following dialysis initiation [5]. The increased risk of cancer in the first year of dialysis may be partially explained by presence of malignancies, without clinical signs, prior to RRT. Indeed, closer medical surveillance of chronic dialysis patients may have resulted in greater detection of tumors.

The analysis according to dialysis modality was limited because of the small number of DNMs in patients receiving peritoneal dialysis (i.e., $n=13 \mathrm{DNMs}$ ). Nonetheless, we documented a statistically significant increased risk of cancer in patients receiving haemodialysis, and an excess risk of borderline statistical significance in the remaining patients. To the best of our knowledge, only one recent investigation compared the two groups directly, showing no significant differences in cancer risk [20].

The present study also highlighted a strongly elevated risk of site-specific cancers. In accordance with other studies $[1-3,14,17]$, a high risk was observed for kidney cancer, probably attributable to the nature of chronic kidney disease (CKD), related urological anomalies or the occurrence of acquired cystic kidney disease [21]. In contrast with other studies [16], we did not find a high 
Table 3 Standardized incidence ratios (SIRs) and 95\% confidence intervals (Cls) in dialysis patients for selected de novo malignancies (DNMs). Friuli Venezia Giulia, north-eastern Italy, 1998-2013

\begin{tabular}{|c|c|c|c|c|}
\hline \multirow[b]{2}{*}{ Cancer type/site } & \multirow[b]{2}{*}{ ICD-9 codes } & \multicolumn{2}{|c|}{ Total $^{a}$} & \multirow[b]{2}{*}{ SIR $(95 \% \mathrm{Cl})$} \\
\hline & & Obs. & Exp. & \\
\hline All but skin non-melanomab & & 249 & 213.7 & $1.17(1.03-1.32)$ \\
\hline Skin non-melanoma & 173 & 93 & 51.3 & $1.81(1.46-2.22)$ \\
\hline Prostate & 185 & 35 & 39.5 & $0.89(0.62-1.23)$ \\
\hline Trachea, bronchus, and lung & 162 & 31 & 28.4 & $1.09(0.74-1.55)$ \\
\hline Colon, rectum, and anus & 153,154 & 27 & 31.7 & $0.85(0.56-1.24)$ \\
\hline Kidney & 189 & 25 & 7.9 & $3.18(2.06-4.69)$ \\
\hline Bladder & 188 & 16 & 17.0 & $0.94(0.54-1.52)$ \\
\hline Oral cavity & $140-149$ & 15 & 6.2 & $2.42(1.36-4.00)$ \\
\hline Stomach & 151 & 14 & 11.6 & $1.20(0.66-2.02)$ \\
\hline Liver & 155 & 14 & 9.2 & $1.53(0.83-2.56)$ \\
\hline Female breast & 174 & 13 & 14.9 & $0.81(0.42-1.41)$ \\
\hline Melanoma & 172 & 9 & 5 & $1.81(0.83-3.43)$ \\
\hline Pancreas & 157 & 7 & 7.5 & $0.93(0.38-1.93)$ \\
\hline Larynx & 161 & 7 & 3.4 & $2.03(0.82-4.18)$ \\
\hline Other and ill-defined sites & 195 & 7 & 1 & $7.31(2.94-15.07)$ \\
\hline Malignant neoplasm without site specification & 199 & 7 & 3.8 & $1.86(0.75-3.84)$ \\
\hline Multiple myeloma & 203 & 5 & 2.9 & $1.72(0.56-4.02)$ \\
\hline Leukemia & $204-208$ & 4 & 4.2 & $0.95(0.26-2.43)$ \\
\hline Corpus uteri & 182 & 4 & 2.1 & $1.94(0.53-4.96)$ \\
\hline Pleura & 163 & 2 & 1.8 & $1.11(0.13-4.00)$ \\
\hline Other and ill-defined sites within the respiratory system & 165 & 2 & 0.6 & $3.14(0.38-11.33)$ \\
\hline Connective and other soft tissue & 171 & 2 & 1.1 & $1.90(0.23-6.87)$ \\
\hline Kaposi's sarcoma & 176 & 2 & 0.2 & $10.29(1.25-37.16)$ \\
\hline Uterus, unspecified & 179 & 2 & 0.3 & $7.0(0.85-25.30)$ \\
\hline Thyroid gland & 193 & 2 & 1.4 & $1.39(0.17-5.01)$ \\
\hline Non-Hodgkin lymphoma & 200,202 & 2 & 6.6 & $0.30(0.04-1.10)$ \\
\hline
\end{tabular}

risk of bladder cancer, which is strongly associated to several diseases that cause ESKD.

Our estimate of Kaposi's sarcoma risk was similar to the findings from a large population-based cohort study [22]. The elevated excess risk emerged for Kaposi's sarcoma is consistent with the evidence that the uremic immune dysfunction status of dialysis patients exposes them to be susceptible to viruses, such as Kaposi's sarcoma-associated herpes virus (KSHV). Moreover, the prevalence of KSHV has been reported to be higher in some Mediterranean countries, particularly in Italy [23, 24]. Accordingly, the high excess risk found for cancers of oral cavity provided further support to the poor immune control of known oncogenic viruses in the ESKD population. Indeed, previous studies have shown higher risks of tongue or mouth cancers [22], which are known to be associated with human papillomavirus. Unfortunately, due to the low number of observed cases in the present investigation, we could not evaluate the SIRs for specific oral cavity subtypes.

NMSC was the most common DNM diagnosed among dialysis patients. Although only a few studies have investigated NMSC during dialysis, our findings confirmed prior evidence [25]. A recent Asian study reported that dialysis patients with uremic pruritus carried a higher risk of NMSC than those without, suggesting that the synergic effect of sun exposure and uremia could be responsible for the carcinogenesis of NMSC [25].

The advanced CKD and side effects of decreased renal function, including impaired function of the immune system and of DNA repair mechanisms as well as chronic infections and inflammations, have been proposed as 
potential promoting factors of cancer [22, 26]. To this regard, the higher risks found in the present study are consistent with the evidence that direct carcinogenic effects of factors related to ESKD are mediated by dialysis treatments [2]. Previous studies have provided limited support to the fact that dialysis may itself increase the risk of cancer [4]. However, it may prolong pre-RRT carcinogenic exposure since it neither completely replaces renal function nor reverses kidney disease [2].

Some limitations of our study are worth noticing. First, as for most studies [16], information about patients' comorbidities -which are highly prevalent in this population- as well as information about etiology for ESKD or lifestyle habits (e.g., smoking, alcohol consumption, or sun exposure) are not routinely collected in the renal registry of the Friuli Venezia Giulia region. Consequently, we could not evaluate their potential confounding effect on the occurrence of cancer in dialysis population. However, this lack of information had limited impact on our aim of quantifying the excess risk of cancer among dialysis patients, in comparison with the corresponding general population. Second, we could not exclude the possibility of overestimation of cancer incidence, as a result of misclassification of prevalent cases as incident ones. However, to minimize this problem we excluded from the analysis the first 30 days of dialysis. A longer lag-time could have been considered between the occurrence and diagnosis of cancer, as we observed the highest excess risk of cancer within the first year. Nevertheless, a sensitivity analysis performed excluding the first 90 days of follow-up showed similar results. Third, the relatively small size of our cohort limited the study power to assess the excess risk of cancer for specific cancer sites/types. Finally, subgroup analysis should be interpreted with caution as the general population cannot be stratified by age at first dialysis, time on dialysis, dialysis modality, and calendar period at first dialysis. As most studies [16], the expected number of cases in each subgroup was calculated according to the incidence rates computed among the average population of Friuli Venezia Giulia region used for the non-stratified analysis. Notwithstanding such limitations, this populationbased study represents the largest cohort to provide an overall picture of cancer risk among dialysis patients in southern Europe. One of the strengths of this study is the complete coverage of Friuli Venezia Giulia dialysis patients thanks to the use of population-based administrative data, which allows a comprehensive assessment of the health status of the whole population of the Friuli Venezia Giulia region. In addition, the use of incidence data collected by the same population-based cancer registry, ensures high standards in terms of quality and completeness of cancer reporting.

\section{Conclusions}

Our findings put in evidence the need of monitoring the cancer burden among ESKD patients undergoing dialysis. The increased risks documented in relation to specific cancer sites and in younger patients suggest that targeted approach to cancer screening need to be implemented in the dialysis population. The link between renal function and cancer risk, and the detection of possible mechanisms through which renal impairment can modulate cancer risk remain a topic of great scientific interest.

\section{Abbreviations \\ Cl: Confidence interval; CKD: Chronic kidney disease; DNM: De novo malignancy; ESKD: End-stage kidney disease; ICD-9: International Classification of Disease, 9th revision; IQR: Interquartile range; KSHV: Kaposi's sarcoma-associated herpes virus; KT: Kidney transplant; NMSC: Non- melanoma skin cancers; RRT: Renal replacement therapies; SIR: Standardized incidence ratio}

\section{Acknowledgements}

The authors wish to thank Mrs. Luigina Mei for editorial assistance. Other members of the Italian Transplant \& Cancer Cohort Study: Sarah Shalaby, Raffaella Petrara, Patrizia Burra, Giacomo Zanus, Stefano Zanini, Paolo Rigotti (Padua University Hospital, Padua, Italy); Maria Rendina, Alfredo Di Leo, Francesco Paolo Schena, Giuseppe Grandaliano, Marco Fiorentino, (University Hospital, Bari, Italy); Augusto Lauro, Antonio Daniele Pinna, Paolo Di Gioia, Sara Pellegrini, Chiara Zanfi, Maria Piera Scolari, Sergio Stefoni, Paola Todeschini, Laura Panicali, Chiara Valentini ("S. Orsola-Malpighi" Hospital, Bologna); Umberto Baccarani, Andrea Risaliti, Gian Luigi Adani, Dario Lorenzin (Udine University, Udine, Italy); Giuseppe Maria Ettorre, Giovanni Vennarecci, Marco Colasanti, Manuela Coco, Fabrizio Ettorre, Roberto Santoro, Lucia Miglioresi (S. Camillo Hospital, Rome, Italy); Francesco Nudo, Massimo Rossi, Gianluca Mennini (Umberto I Policlinic, Rome, Italy); Luca Toti, Giuseppe Tisone, Annachiara Casella, Laura Fazzolari, Daniele Sforza, Giuseppe laria, Carlo Gazia, Chiara Belardi (Tor Vergata University, Rome, Italy); Claudia Cimaglia, Alessandro Agresta, Gianpiero D'Offizi, Ubaldo Visco Comandini, Raffaella Lionetti, Marzia Montalbano, Chiara Taibi (INMI "L. Spallanzani", Rome, Italy); Giovanni Fantola, Fausto Zamboni, Gian Benedetto Piredda, Maria Benigna Michittu, Maria Gavina Murgia, Bruno Onano ("Brotzu" Hospital, Cagliari, Italy); Lucia Fratino, Luigino Dal Maso, Paolo De Paoli, Diana Verdirosi, Emanuela Vaccher (IRCCS CRO Aviano National Cancer Institute, Aviano, Italy); Francesco Pisani, Antonio Famulari, Federica Delreno, Samuele lesari, Linda De Luca (University of L'Aquila, L'Aquila, Italy); Maurizio Iaria, Enzo Capocasale, Elena Cremaschi (Parma University Hospital); Silvio Sandrini, Francesca Valerio, Valentina Mazzucotelli, Nicola Bossini, Gisella Setti (Spedali Civili of Brescia, Brescia, Italy); Massimiliano Veroux, Pierfrancesco Veroux, Giuseppe Giuffrida, Alessia Giaquinta, Domenico Zerbo (Policlinico of Catania, Catania, Italy); Ghil Busnach, Laura Di Leo, Maria Luisa Perrino, Marialuisa Querques, Valeriana Colombo, Maria Chiara Sghirlanzoni ("Niguarda Ca' Granda" Hospital, Milan, Italy); Piergiorgio Messa, Antonio Leoni ("Maggiore-Mangiagalli "Hospital, Milan, Italy); Laura Galatioto, Salvatore Gruttadauria, Vito Sparacino, Flavia Caputo, Barbara Buscemi (Policlinico of Palermo, Palermo, Italy); Franco Citterio, Gionata Spagnoletti, Maria Paola Salerno, Evaldo Favi (Policlinico "A. Gemelli", Rome, Italy); Giuseppe Paolo Segoloni, Luigi Biancone, Antonio Lavacca ("Molinette" Hospital, Turin, Italy); Maria Cristina Maresca, Carmelo Cascone, Bice Virgilio (Treviso Hospital, Treviso, Italy); Donato Donati, Fiorella Dossi, Andrea Fontanella, Andrea Ambrosini, Marco Di Cicco ("Ospedale di Circolo", Varese, Italy).

\section{Funding}

This work was funded by the Italian Association for Research on Cancer (AIRC IG No. 19112). The funding body had no role in the study design, in the data collection, analysis, and interpretation, and in the manuscript writing. 


\section{Availability of data and materials}

The study dataset is available upon request for research purposes only, under a data transfer agreement, from the Unit of Cancer Epidemiology, IRCCS CRO Aviano National Cancer Institute.

\section{Authors' contributions}

DS conceived the study; MT and DS drafted the manuscript; MT and FT conducted the statistical analyses; FT and SDZ participated in the acquisition of incidence data and population data; EC, LF, PP, FC, LZ, and GB provided support in the interpretation of results. All the authors have critically revised the manuscript for important intellectual content and have given final approval of the version to be published.

\section{Ethics approval and consent to participate}

Cancer Registries are identified as collectors of personal data for surveillance purposes without the need of explicit individual consent. The approval of a research ethic committee is not required as this descriptive study was conducted without any direct or indirect intervention on patients.

\section{Consent for publication}

Not applicable.

\section{Competing interests}

The authors declare that they have no competing interests.

\section{Publisher's Note}

Springer Nature remains neutral with regard to jurisdictional claims in published maps and institutional affiliations.

\begin{abstract}
Author details
${ }^{1}$ Cancer Epidemiology Unit, Centro di Riferimento Oncologico di Aviano (CRO) IRCCS, via Franco Gallini 2, 33081 Aviano, (PN), Italy. ${ }^{2}$ Azienda Regionale di Coordinamento per la Salute, Udine, Italy. ${ }^{3}$ Kidney and Pancreas Transplantation Unit, Padua University Hospital, Padua, Italy. ${ }^{4}$ Department of Epidemiology and Pre-Clinical Research, National Institute for Infectious Diseases "L. Spallanzani", Rome, Italy. ${ }^{5}$ Renal Transplantation Unit, Department of Surgical Science, Università Cattolica Sacro Cuore, Rome, Italy. ${ }^{6}$ Azienda Sanitaria Universitaria Integrata di Trieste, Trieste, Italy.
\end{abstract}

Received: 28 November 2018 Accepted: 7 March 2019

Published online: 28 March 2019

\section{References}

1. Maisonneuve P, Agodoa L, Gellert R, Stewart JH, Buccianti G, Lowenfels AB, et al. Cancer in patients on dialysis for end-stage renal disease: an international collaborative study. Lancet. 1999;354:93-9.

2. Stewart JH, Vajdic CM, van Leeuwen MT, Amin J, Webster AC, Chapman JR, et al. The pattern of excess cancer in dialysis and transplantation. Nephrol Dial Transplant. 2009;24:3225-31.

3. Lin H-F, Li Y-H, Wang C-H, Chou C-L, Kuo D-J, Fang T-C. Increased risk of cancer in chronic dialysis patients: a population-based cohort study in Taiwan. Nephrol Dial Transplant. 2012;27:1585-90.

4. Wong G, Turner RM, Chapman JR, Howell M, Lim WH, Webster AC, et al. Time on Dialysis and Cancer risk after kidney transplantation. Transp J. 2013; 95:114-21.

5. Lin MY, Kuo MC, Hung CC, Wu WJ, Chen LT, Yu ML, et al. Association of Dialysis with the risks of cancers. PLoS One. 2015;10(4):e0122856.

6. Lee JE, Han SH, Cho BC, Park JT, Yoo TH, Kim BS, et al. Cancer in patients on chronic Dialysis in Korea. J Korean Med Sci. 2009;24(Suppl 1):S95-101.

7. Butler AM, Olshan AF, Kshirsagar AV, Edwards JK, Nielsen ME, Wheeler SB, et al. Cancer incidence among US Medicare ESRD patients receiving hemodialysis, 1996-2009. Am J Kidney Dis. 2015:65:763-72.

8. Schupp N, Heidland A, Stopper H. Genomic damage in Endstage renal disease-contribution of uremic toxins. Toxins (Basel). 2010;210:2340-58.

9. Hortlund M, Mühr LSA, Storm H, Engholm G, Dillner J, Bzhalava D. Cancer risks after solid organ transplantation and after long-term dialysis. Int J Cancer. 2017;140:1091-101.

10. Pontoriero G, Pozzoni P, Vecchio LD, Locatelli F. International study of health care organization and financing for renal replacement therapy in Italy: an evolving reality. Int J Health Care Finance Econ. 2007;7:201-15.
11. Mazzucotelli V, Piselli P, Verdirosi D, Cimaglia C, Cancarini G, Serraino D, et al. De novo cancer in patients on dialysis and after renal transplantation: North-Western Italy, 1997-2012. J Nephrol. 2017;30:851-7.

12. Hosmer D, Lemeshow S, May S. Applied survival analysis: regression modeling of time-to-event data, Second Edition. Hoboken: John Wiley \& Sons; 2008.

13. Breslow NE, Day NE. Statistical methods in cancer research Vol. I: the analysis of case-controls studies. IARC scientific publication no 32. Lyon: International Agency for Research on Cancer; 1980.

14. Cheung CY, Chan GCW, Chan SK, Ng F, Lam MF, Wong SSH, et al. Cancer incidence and mortality in chronic Dialysis population: a multicenter cohort study. Am J Nephrol. 2016;43:153-9.

15. Birkeland $\mathrm{SA}$, Løkkegaard $\mathrm{H}$, Storm HH. Cancer risk in patients on dialysis and after renal transplantation. Lancet. 2000;355:1886-7.

16. Shang W, Huang L, Li L, Li X, Zeng R, Ge S, et al. Cancer risk in patients receiving renal replacement therapy: a meta-analysis of cohort studies. Mol Clin Oncol. 2016:5:315-25.

17. Loy EY, Choong HL, Chow KY. Cancer among end-stage renal disease patients on Dialysis. Ann Acad Med Singap. 2013;42:640-5.

18. Heidland A, Bahner U, Vamvakas S. Incidence and spectrum of dialysisassociated cancer in three continents. Am J Kidney Dis. 2000;35:347-51.

19. Chien C-C, Han M-M, Chiu Y-H, Wang J-J, Chu C-C, Hung C-Y, et al. Epidemiology of cancer in end-stage renal disease dialysis patients: a national cohort study in Taiwan. J Cancer. 2017:8:9-18.

20. Lee $Y$, Hung $S$, Wang $H$, Lin $C$, Wang $H$, Chang $M$, et al. Is there different risk of cancer among end-stage renal disease patients undergoing hemodialysis and peritoneal dialysis? Cancer Med. 2018;7(2):485-98.

21. Satoh S, Tsuchiya N, Habuchi T, Ishiyama T, Seimo K, Kato T. Renal cell and transitional cell carcinoma in a Japanese population undergoing maintenance dialysis. J Urol. 2005;174:1749-53.

22. Vajdic CM, McDonald SP, McCredie MRE, van Leeuwen MT, Stewart JH, Law $M$, et al. Cancer incidence before and after kidney transplantation. JAMA. 2006:296:2823-31

23. Piselli P, Serraino D, Segoloni GP, Sandrini S, Piredda GB, Scolari MP, et al. Risk of de novo cancers after transplantation: results from a cohort of 7217 kidney transplant recipients, Italy 1997-2009. Eur J Cancer. 2013;49:336-44.

24. García-Astudillo LA, Leyva-Cobián F. Human herpesvirus-8 infection and Kaposi's sarcoma after liver and kidney transplantation in different geographical areas of Spain. Transpl Immunol. 2006:17:65-9.

25. Wang CC, Tang CH, Wang CY, Huang SY, Sue YM. Risk of skin cancer in patients on chronic haemodialysis: a nationwide, population-based study in Taiwan. Br J Dermatol. 2016:175:1175-82.

26. Vamvakas S, Bahner U, Heidland A. Cancer in end-stage renal disease: potential factors involved. Am 」 Nephrol. 1998:18:89-95.

Ready to submit your research? Choose BMC and benefit from

- fast, convenient online submission

- thorough peer review by experienced researchers in your field

- rapid publication on acceptance

- support for research data, including large and complex data types

- gold Open Access which fosters wider collaboration and increased citations

- maximum visibility for your research: over $100 \mathrm{M}$ website views per year

At BMC, research is always in progress.

Learn more biomedcentral.com/submissions 